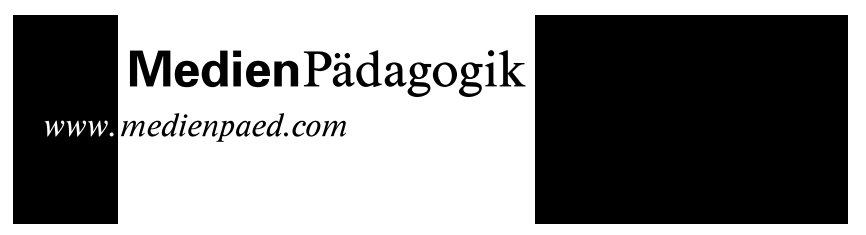

Michael Kerres und Claudia de Witt

8.11 .2002

\section{Quo vadis Mediendidaktik? Zur theoretischen Fundierung von}

\section{Mediendidaktik}

\section{Einleitung}

Die mediendidaktische Forschung ist geprägt durch Einzelprojekte, in denen die Möglichkeiten neuer Lernmedien erprobt werden. Zur theoretischen Begründung und Einordnung der Vorhaben werden in der Regel die lerntheoretischen Ansätze des Behaviorismus, Kognitivismus und Konstruktivismus herangezogen. Im Folgenden sollen einige Probleme einer solchen Fundierung von Mediendidaktik diskutiert werden. Die bisherige Theoriebildung in der Mediendidaktik ist durch die Suche nach dem «einen» besten Ansatz des Lernens und Lehrens geprägt. Perspektivisch wären jedoch lerntheoretische und bildungstheoretische Ansätzen stärker zu verknüpfen. Dabei kann der Pragmatismus sensu Dewey als richtungsweisende Grundlage zur theoretischen Fundierung von Mediendidaktik dienen.

\section{Der «Behaviorismus» als Theorie des computergestützten Lernens}

Die zentralen theoretischen Konzepte der Mediendidaktik sind bislang vorrangig der lerntheoretischen Diskussion entlehnt. Zunächst wäre hier die Position des Behaviorismus zu nennen, der in den 60er Jahren als theoretisches Rahmenmodell zur Fundierung der ersten Generation computergestützter Lernprogramme diente (vgl. Pagliaro 1983; Dick 1987; Reiser 1987; Benjamin 1988), und heute in der Regel als Gegenpol zu einer «konstruktivistischen Didaktik» wahrgenommen wird. B. F. Skinner sah in der neuen Technik interessante Anwendungsmöglichkeiten für seine theoretischen Überlegungen.

Wesentliche Aussagen eines behavioristischen Ansatzes zur Gestaltung von Lernangeboten lauten:
- Lernen wird durch Reaktionen der Umwelt beeinflusst. Lernen erfordert Rückmeldung über den Lernfortschritt und zwar so unmittelbar, wie für den Lernprozess erforderlich.

- Lernen erfordert Aktivität des Lerners, der Konsum präsentierter Informationen ist nicht hinreichend.

- Lernen braucht Erfolgserlebnisse, damit Lerner aktiv bleiben.

- Erfolgserlebnisse können nur stattfinden, wenn der Lerner mit Lernmaterial konfrontiert wird, das seinem aktuellen Lernstand entspricht.

- Lerninhalte sind in kleine Lerneinheiten zu zergliedern, damit ihre Präsentation an den aktuellen Lernstand optimal angepasst werden können.

Über diese Aussagen kann man streiten, gleichwohl ist ersichtlich, dass sie keineswegs so abwegig sind, wie in der «Konstruktivismus-Debatte» teilweise nahe gelegt worden ist. Im Kontext eines behavioristischen Ansatzes werden ein kleinschrittiges Vorgehen mit enger Führung des Lerners durch den Computer und regelmässige Rückmeldungen favorisiert. Der Ansatz bot eine seinerzeit weithin überzeugende theoretische Fundierung, der das computerbasierte Lernen weltweit popularisierte. Allerdings erscheint genau diese theoretische Fundierung insofern fragwürdig, als es sich - bis heute - bei den entsprechenden CBTs kaum um eine konsequente «Anwendung» behavioristischer Theorie handelt, sondern vielmehr um eine eher vage, von behavioristischen Konzepten «inspirierte» Konstruktion, die bis heute zu Missverständnissen führt.

Die verschiedentlich vorgebrachte, kritische Frage lautet, ob bei der Programmierten Instruktion nach SKINNER überhaupt das Prinzip des Verstärkungslernens Anwendung findet? Behavioristische Lerntheorien untersuchen beobachtbares Verhalten und wie Reaktionen der Umwelt auf die Auftretenswahrscheinlichkeit dieses Verhalten wirken: Wird ein konkretes Verhalten in Zukunft häufiger, schneller, stärker etc. auftreten? Damit stellt sich die Frage, welches Verhalten beim computergestützten Lernen eigentlich verstärkt werden kann: Ist es das Eintippen von Buchstaben oder das Arbeiten an einem Rechner? Lässt sich die Aneignung von Wissen «verstärken»?

Nach SKINNER werden die richtigen Reaktionen auf Fragen verstärkt. Doch das Ziel der Bearbeitung eines Lernprogramms besteht kaum darin, die richtigen Reaktionen auf bestimmte Fragen zu erlernen. Es besteht vielmehr die Hoffnung, dass sich Wissen aufbaut, das auch bei anderen 
Fragen oder in anderen Kontexten abgerufen und genutzt werden kann Würde das gesamte Wissen aus «richtigen Reaktionen auf Fragen» bestehen, so wären Personen nie in der Lage, auf immer neue, unerwartete Situationen schnell, flexibel und (manchmal auch) kreativ zu reagieren. Wissen ist nur schwer als Summe der erlernten Verhaltensketten beschreibbar, bei denen für bestimmte Situationen, Anforderungen, Fragen etc. die richtigen Reaktionen, Antworten, Verhaltensweisen etc. abgespeichert sind, denen auf der Grundlage der individuellen Lernerfahrung jeweils Erfolgswahrscheinlichkeiten zugeordnet sind. Bereits HILGARD (1964) kritisierte, dass höchstens vage Analogien zwischen behavioristischen Lernmechanismen und den Lernprogrammen bestünden, deren Funktionsweise kaum mit Prinzipien des Verstärkungslernens $\mathrm{zu}$ erklären sind. Mit der «Bildungskybernetik» lag im übrigen seinerzeit ein vor allem im deutschsprachigen Raum diskutiertes alternatives theoretisches Modell zur Erklärung der Programmierten Instruktion vor, das bestimmte Annahmen späterer kognitiver Ansätze vorwegnahm (vgl. Frank/ Meder 1971; Cube von 1980; Frank 1996).

Kaum haltbar ist jedoch die vielfach anzutreffende Behauptung, dass eine behavioristische Konzeption von Lernmedien ein «passives Lernen» implizieren würde. Denn wenig lag Skinner mehr am Herzen als das aktive Lernen zu forcieren; immer wieder kritisierte er, wie wenig aktiv Lernende im konventionellen Unterricht sind. Es wird von der Theorie des operanten Konditionierens gesprochen, und damit von der Konditionierung von Verhalten, das von der Person ausgeht und nicht, wie beim respondenten Konditionieren als Reflex auf die Umwelt. Gelernt werden kann nach Skinner überhaupt nur, wenn eine Person aktiv wird, jeder Versuch Organismen durch Informationen zu belehren muss scheitern, solange der Organismus nicht unmittelbar durch eigenes Operieren von der Umwelt eine Reaktion erfährt.

Wie lässt sich dann überhaupt erklären, dass der Behaviorismus in der wissenschaftlichen Diskussion heute oft mit «passivem Lernen» verbunden wird? Dies hängt vielleicht damit zusammen, dass die seinerzeit entwickelten Lehrmaschinen technisch sehr eingeschränkte Möglichkeiten für «Aktivitäten» der Lernenden vorsahen: Mit den Multiple - Choice Fragen, über deren Auswertung der weitere Verlauf des Programms gesteuert wird, reduziert sich die Aktivität des Lernenden auf die Auswahl von Antwortalternativen per Tastendruck. Skinner lehnte dies aus theoretischer Sicht übrigens immer ab. Er forderte Freitextantworten und zwar mit der Begründung, dass bei Multiple - Choice - Fragen den Lernenden neben einer richtigen auch falsche Antwortalternativen präsentiert werden (müssen), die sich möglicherweise einprägen. Heute würden wir die «operanten» Aktivitäten von Lernenden bei Anwendung von Skinners Überlegungen in ganz anderen technischen Szenarios untersuchen wollen: in dem Umgang mit virtuellen Welten und Labors, mit Computersimulationen und VR-Systemen. Hier liesse sich sehr wohl untersuchen, wie operantes Verhalten in diesen Lernumgebungen auf der Basis behavioristischer Modelle erklärt werden könnte.

Was Skinner tatsächlich nicht behandelte, sondern programmatisch ausblendete, ist die innerpsychische Verarbeitung der Informationen und Umweltreize. Auf diese Weise konnte er nicht erklären, wie Menschen komplexe Wissensstrukturen und Problemlösungen entwickeln. So erscheint Lernen als ein rein umweltgetriebener, mechanischer Prozess, dem der Einzelne durch Umweltkontingenzen «ausgeliefert» ist. Dabei muss diese Position, die sich rein auf äusseres, beobachtbares Verhalten konzentriert und alle psychischen Prozesse als black box ausklammert, vor allem als Gegenbewegung zu der seinerzeit vorherrschenden rein spekulativen Bewusstseinspsychologie aufgefasst werden. Was Skinner durch diese Prämisse tatsächlich nicht thematisieren konnte, ist der Erwerb komplexerer geistiger Fertigkeiten und Konzepte und wie die Umwelt dies unterstützen kann. Das Modell konnte letztlich nur den Erwerb elementarer Verhaltensweisen erklären.

Die beschriebenen Unstimmigkeiten in der Rezeption des Behaviorismus erscheinen recht offensichtlich. Die kollektive Reinterpretation des behavioristischen Paradigmas im Sinne des «passiven Lernens» in der im Folgenden beschriebenen Konstruktivismus-Debatte scheint somit eher als gruppendynamisches Phänomen erklärbar, eignet sich «der Behaviorismus» doch hervorragend als Folie, gegen die die Vorzüge der «neuen» Position aufgebaut werden kann. «Behaviorismus» scheint als sozial negativ besetztes Konzept hierfür besonders geeignet, nicht zuletzt weil es kaum einen ernsthaften Verfechter des Behaviorismus mehr gibt. Die Ablehnung des «Behaviorismus» ist damit in der wissenschaftlichen Diskussion wenig «gefährlich», im Gegenteil sie sichert soziale Akzeptanz in der scientific community.

Wir können also im Wesentlichen zwei Probleme in der Rezeption des behavioristischen Paradigmas in der Mediendidaktik identifizieren: Zum einen fällt auf, dass die verbreitete Re-Konstruktion des behavioristischen 
Paradigmas mit den Intentionen zur Aktivität bzw. Passivität des Lernens nicht übereinstimmen. Zum anderen bleibt unsicher, ob die Anwendung der entsprechenden Prinzipien in den frühen Lehrmaschinen tatsächlich gelungen ist, und ob nicht gerade neuere Anwendungen aus dem Bereich Computersimulation / -spiele und VR interessante Anknüpfungspunkte für eine erneute und vorurteilslose Beschäftigung mit Konzepten des Behaviourismus bieten.

\section{Zur gesellschaftlichen Konstruktion der «Dialogsysteme»}

Die frühe Diskussion über Computer und Programmierte Instruktion war durch den die Lerntheorie seinerzeit dominierenden Behaviorismus geprägt. Im Folgenden wollen wir jedoch aufzeigen, dass mehr noch als diese - zum Teil falsch interpretierte - lerntheoretische Konzeption vor allem die gesellschaftliche Konstruktion des Computers als Dialogsystem die Vorstellungen der didaktischen Computernutzung beeinflusst haben.

Die ersten Versuche zum computergestützten Lernen griffen die seinerzeit neuen Möglichkeiten von Rechnern auf, im «Dialogbetrieb» mit dem Benutzer dessen Eingaben direkt - statt in einer Warteschlange («Stapelbetrieb») zu verarbeiten. Mit dieser neuen Form der nunmehr «interaktiv» arbeitenden Systeme ergab sich die faszinierende Möglichkeit, Programme für «Dialoge» zwischen Gerät und Benutzer zu entwickeln. Es lag nahe, diese «Dialoge» nicht nur für die «interaktive» Systembedienung zu nutzen, sondern auch zu prüfen, inwieweit diese «Dialoge» sich auf andere Anwendungskontexte, etwa den pädagogischen Bereich, ausweiten liessen. Die Herausforderung bestand also darin, die Reichweite computerbasierter «Dialogfähigkeit» in der Mensch-Maschine-«Interaktion» zu eruieren. Der Einsatz der Technik für «pädagogische Dialoge» bot sich gerade im universitären Umfeld - als Forschungsgegenstand an.

Dies alles musste für Erziehungswissenschaftler/innen befremdlich wirken, hatten doch die hier teilweise programmatisch, teilweise naiv und teilweise auch provozierend gebrauchten Begriffe «Dialog», «Kommunikation» und «Interaktion» in der geistes- und sozialwissenschaftlichen Tradition - gerade in Deutschland - einen ganz anderen Bedeutungshorizont. Denn dort sind diese Begriffe mit Grundfragen menschlicher und gesellschaftlicher Existenz verbunden. ${ }^{1}$ Ein offensichtlich harter Kontrast zu den läppisch

\footnotetext{
1 Vgl. z. B. das dialogische Prinzip bei Buber, die Kommunikative Didaktik bei Schäfer/Schaller oder der Interaktionsbegriff bei G. H. Mead.

wirkenden Computer-«Dialogen», die sich auf die Ausgabe von Zeichen auf einem Bildschirm infolge der manuellen Betätigung von Tasten beschränkten.

Die hier zugrunde liegende und bis heute fortdauernde Konfrontation hängt ganz wesentlich mit der teilweise masslos übertriebenen, wenig reflektierten Begriffsverwendung seitens der Informatik zusammen, die den qualitativen Unterschied zwischen dem, was zwischen Mensch und Maschine passiert, und zwischenmenschlicher Dialogfähigkeit ignoriert. Es wird davon ausgegangen, dass sich alle Kommunikationsvorgänge im Wesentlichen nur quantitativ unterscheiden. Es handelt sich um lediglich unterschiedlich komplexe Varianten von «Dialog», «Kommunikation» und «Interaktion». Wenn man ein technisches System dazu bringen kann auf die Betätigung einer Taste hin eine Bildschirmausgabe zu erzeugen, dann wird man - hinreichend Forschung und Forschungsförderung vorausgesetzt - auch (irgendwann) in der Lage sein, «intelligentere» Formen der «Kommunikation» nachzubilden, wie sie zwischen Menschen statt findet. Die Marketingleistung der Informatik(industrie) bestand darin, die Begrifflichkeit für originär zwischenmenschliche Phänomene auf technische Systeme zu übertragen und damit das technische System semantisch aufzuwerten und zu überhöhen. Erst diese semantische «Aufladung» transformiert den banalen «Rechner» zur zentralen Kategorie der «digitalen Informationsund Wissensgesellschaft».

Diese Aufladung und ihre ubiquitäre Verbreitung haben jedoch gleichzeitig dazu beigetragen, dass genau diese Begriffe im gesellschaftlichen Diskurs dann aber an Wertigkeit verlieren. Beinhaltete Kommunikation in der «Kommunion» noch die Teilhabe am Göttlichen, so wird der Begriff heute zunehmend für bereits weitgehend triviale Umstände genutzt: wir kommunizieren mit dem Fernseher oder einem Drucker, wir kommunizieren eine Meinung, eine Botschaft ... Auch «dialogfähig» kann heute schon ein Fernseher oder ein Drucker sein. Da hatten die Geistes- und Sozialwissenschaftler Buber, Habermas, Apel, oder Schäfer/Schaller doch eine andere Vorstellung von Kommunikation und Dialog.

Auf der anderen Seite findet sich gerade in der deutschsprachigen Erziehungswissenschaft eine besondere Sensibilität gegenüber einer solch simplifizierenden und reduktionistischen Sicht auf diese im Kontext von Bildung und Erziehung zentralen Begriffe. Es musste die Erziehungswissenschaft herausfordern, für die der qualitative Unterschied zwischen dem Austausch von Zeichen einerseits und der menschlichen Dialog- 
fähigkeit anderseits offensichtlich ist: Der blosse Austausch von Zeichen ist für den Menschen belanglos. Die eigentliche Leistung des Menschen ist der Dialog, der es zum Beispiel möglich macht, dass Menschen Konsens finden, Frieden schliessen, und der dazu beitragen kann, dass das Überleben der Menschheit möglich wird. Für eine Erziehungswissenschaft, die ihr pädagogisches Anliegen eben nicht auf den optimierten «Austausch von Informationen» reduzieren will, sondern traditionell Perspektiven von Humanität, Solidarität oder Gerechtigkeit vertritt, sind die genannten Implikationen der typischen Begriffsnutzung in der Informatik nicht nur problematisch, sondern dem Anliegen der Erziehungswissenschaft geradezu gegenläufig.

In der US-amerikanischen Erziehungswissenschaft ist dieser Horizont, der mit dem Begriff der «Bildung» verbunden ist, im Ganzen nicht so präsent wie in Deutschland. In den USA beschäftigte man sich in der Erziehungswissenschaft immer schon stärker mit Fragen der Qualifikation und Qualifizierung, während die deutschsprachige Pädagogik den qualitativen Unterschied zwischen Bildung einerseits und Ausbildung und Training anderseits immer besonders betont hat.

Auf diese Weise hat sich in Deutschland - pointiert formuliert - eine besondere, bis heute andauernde Kluft zwischen einer typisch informatischen Sicht entwickelt, die eine wenig reflektierte Begrifflichkeit unter Vernachlässigung deren wissenschaftlichen Kontext und Tradition pflegte, und einer erziehungswissenschaftlichen Sicht, die diese Entwicklung mit durchweg fundamentaler Skepsis und Distanz beobachtete.

In den 70er Jahren stellte sich schnell heraus, dass die bisherigen computerbasierten Lernprogramme wenig Akzeptanz finden würden. Ihr monotoner Ablauf und die Zerlegung komplexer Sinneinheiten in kleine Lerneinheiten stiessen im pädagogischen Feld schnell an ihre Grenzen. Verglichen mit den öden und dumpfen «Computerdialogen» erschien Unterricht selbst der schlechtesten Lehrkraft einfühlsam und differenziert. Das Postulat der frühen Protagonisten, Unterricht $\mathrm{zu}$ optimieren und besser an den Einzelnen anzupassen, erschien wie Hohn. Die Herausforderung, wie sie nun von der Mehrheit der neuen Forschungsaktivitäten im Kontext der «Künstlichen Intelligenz» gesehen wurde, bestand darin, mithilfe anderer Softwaretechniken Computerdialoge zu programmieren, die sich durch deutlich mehr «Intelligenz» auszeichnen würden. Der Kognitivismus verfolgte damit das ursprüngliche Anliegen von SKINNER u. a. mit verfeinerten technischen Lösungen weiter.
Mit «intelligenten tutoriellen Systemen» wurde in den 80er Jahren untersucht, wie aus Benutzereingaben ein Modell der Kompetenz des Lernenden erstellt werden kann, auf dessen Basis differenziertere Lernangebote generiert werden können. Die Forschung machte schnell deutlich, wie komplex ein solches Unterfangen ist. Die mühsamen Versuche hierzu versandeten schnell als man erkannte, dass selbst für kleinste Wissensausschnitte und einfache kognitive Fertigkeiten langwierige Verfahren zu entwickeln sind. Und selbst die zuverlässige Diagnose von Lerndefiziten stellt keineswegs sicher, dass ein «gutes» Lernangebot entsteht (vgl. Beiträge hierzu in: Sleeman/ Brown 1982; Anderson/ Boyle et al. 1985; Mandl/ Lesgold 1988; Polson/ Richardson 1988).

Insgesamt setzten sich die Überschätzung künstlicher Intelligenz und die Unterschätzung natürlicher Intelligenz fort. Für die Bildungspraxis hatten diese Arbeiten insgesamt wenige Auswirkungen. Wenig ernsthaft wurde überlegt, ob das Versagen der ersten Versuche auch daran gelegen haben könnte, dass der Computertechnik eine problematische Funktion im Lernprozess zugeschrieben wurde, und dass die Konzeption des computerunterstützten Lernens und nicht (nur) deren technische Umsetzung möglicherweise anderer Ansätze bedarf.

Die Grenzen des computerbasierten Lernens wurden in den 80er Jahren damit deutlicher und in der damit einsetzenden bildungstechnologischen Krise wurde der Computer für Lernzwecke immer mehr in Frage gestellt. Erst Mitte der 90er Jahre setzte ein Umschwung ein, der das Interesse am computergestützten Lernen erneut belebte. Dies hatte zunächst mit dem Aufkommen der Multimedia-Technologie zu tun; ganz wesentlich erscheint uns jedoch auch eine zunehmend andere Sichtweise von Computern.

\section{Das Paradigma des Konstruktivismus}

In den 90er Jahren veränderte sich die gesellschaftliche Wahrnehmung des Phänomens «Computer»: In der Frühphase wurden Computer als quasiintelligentes Pendant des Menschen, mit dem Menschen «kommunizieren» und «Dialoge» führen, oder gar als intelligente Nachbildung des Menschen wahrgenommen. Die zunehmende Verbreitung der Technik in der Arbeitsund Lebenswelt der Menschen dekonstruierte diese Überhöhung. Aus dialogfähigen Systemen wurden Werkzeuge, die Menschen für ihre Aufgaben und Interessen nutzen. Aus der Bedienung von Systemen wurde die Benutzung von Werkzeugen. 
Diese veränderte Sicht des Computers hing mit neuen technischen Möglichkeiten zusammen, die einen anderen Umgang mit Computern ermöglichten (grafische Benutzeroberflächen, multimediale Information etc.). Benutzer/innen müssen sich zunehmend weniger schwer verständlichen Systemdialogen «unterwerfen», sondern erwarten eine intuitive Benutzeroberfläche, die bei Arbeitsprozessen optimale Unterstützung bietet Dieser Wandel erst ermöglichte die massenhafte Verbreitung des Computers und seine Akzeptanz als universelles Werkzeug der Wissenserschliessung und -kommunikation zu Ende der 90er Jahre.

Was sich u. E. in der Zeit wesentlich verändert hat, ist die gesellschaftliche Konstruktion des Artefakts «Computer». In der Mediendidaktik ging hiermit einher die Suche nach Konzepten des Computereinsatzes für LehrLernzwecke, die auf diese veränderte Wahrnehmung und Nutzung eingingen. Der «Konstruktivismus» lieferte dazu einen passenden Theoriehintergrund, indem er den Benutzer als aktiv konstruierendes Wesen im sozialen Kontext darstellte. Besonderes Anliegen war die Überwindung von «trägem Wissen», d. h. Wissen, welches möglicherweise in einer Prüfung abrufbar ist, aber in realen Anforderungen nicht angewendet werden kann.

Folgende Aussagen charakterisieren eine typisch konstruktivistische Auffassung des Lernens (Dubs 1995; Duit 1995; Gerstenmaier/ Mandl 1995; Gerstenmeier/ Mandl 1999a; Glasersfeld 1999; vgl. Rustemeyer 1999; Siebert 1999):

- Lernen basiert auf der eigenständigen, aktiven Auseinandersetzung eines Individuums.

- Lerninhalte sind in grössere, sinnhafte Einheiten zu strukturieren, damit der Lernstoff im Zusammenhang erfasst werden kann.

- Lernen geschieht in der aktiven Auseinandersetzung mit komplexen, authentischen Aufgabenstellungen.

- Lernförderlich ist die Konfrontation mit verschiedenen Sichten eines Problems.

- Lernen erfordert die Einbettung in einen sozialen Kontext und die Teilhabe an einer Gemeinschaft von Experten und Expertinnen.

Ein konstruktivistischer Ansatz in der Didaktik betont die Auseinandersetzung mit ganzheitlichen, übergreifenden Aufgaben. Der entscheidende Unterschied zu behavioristischen Konzepten besteht in der Ablehnung der analytisch-zergliedernden Aufbereitung und kleinschrittigen Präsentation der Lehrinhalte. Darüber hinaus wird der soziale Bezug des Lernens betont:
Lernen in Kooperation mit Anderen, im unmittelbaren Kontakt zu Experten und Expertinnen, in einem Kontext, der realitätsnahe Erfahrungen ermöglicht. Die lehrende Instanz offeriert Lernangebote und unterstützt die Lernenden, aber nur soviel wie für den Lernfortschritt erforderlich. Im behavioristischen Konzept greift die lehrende Instanz dagegen stärker ein, idealer Weise steuert und regelt sie den Lernprozess vollständig, um das Lehrziel optimal und effizient zu erreichen.

Mithilfe digitaler Multimedien liessen sich manche konstruktivistische Prinzipien besonders gut umsetzen und erproben, wie z. B. die Präsentation von realitätsnah dargestellten Fällen, das kooperative Lernen in Lerngemeinschaften, das aktive Explorieren in nachgebildeten Umgebungen und manches mehr. Deswegen erzielten die neuen technischen Entwicklungen im Multimedia-Sektor besondere Aufmerksamkeit, wie zum Beispiel:

- Hypertext-/Hypermedia-Systeme, bei denen Lernende in nicht-linearen Medien - etwa im Internet - navigieren, dabei aktiv Informationen nach ihren Interessen wählen und so ihren eigenen Lernweg suchen,

- fallbasierte Systeme, bei denen Lernende mit «authentischen» Fälle aus der Praxis konfrontiert werden (z. B. Diagnosestellung in der Medizin, Entscheidungsfindung in der Betriebswirtschaft),

- Computersimulationen, virtuelle Labore oder virtual realityAnwendungen, bei denen Fertigkeiten im Umgang mit ab-/nachgebildeten Systemen erworben werden,

- Ansätze der produktiven Medienarbeit, bei denen Lernende alleine oder in Gruppen Medien produzieren, - etwa im Internet - publizieren und zur Diskussion stellen,

- kooperative Lernumgebungen, die auf Groupware oder ähnlichen Umgebungen basieren, bei denen Lernende gemeinsam an Lernaufgaben und Projekten arbeiten (CSCL).

Die Debatte über Konstruktivismus in der Erziehungswissenschaft betrifft eine ganze Reihe von Fragen, z. B. ob tatsächlich von einer «konstruktivistischen Didaktik» gesprochen werden kann oder sollte und ob es sich um ein hinreichend neues und abgrenzungsscharfes («paradigmatisches») Konzept handelt. Im Hinblick auf die didaktischen Modellvorstellungen muss konstatiert werden, dass es sich hier im Wesentlichen um tatsächlich altbekannte Ansätze handelt, wie sie z. B. in reformpädagogischen Initiativen zu Beginn des 20. Jahrhunderts formuliert worden sind. Hierbei sollen nicht die grundlegenden erkenntnistheoretischen und epistemiolo- 
gischen Unterschiede der Positionen geleugnet oder geschmälert werden. Es geht hier alleine um die Frage der Implikationen für die theoretische Fundierung einer Mediendidaktik.

In der erziehungswissenschaftlichen Diskussion hat sich der «Konstruktivismus» als überraschend wenig präzises Konzept herausgestellt. Anders als etwa im Kontext von Wissenschaftstheorie oder Wahrnehmungspsychologie ist das Spezifische eines konstruktivistischen Ansatzes in der Didaktik - trotz des häufigen Bezugs hierauf - schwer zu fassen. Der theoretische Bezug einer «konstruktivistischen Didaktik» zu verschiedenen Theorieansätzen der Erkenntnis-, Wissenschaftstheorie, Psychologie, Neurologie etc. stellt sich als relativ vage heraus (vgl. ausführlicher z. B. Rustemeyer 1999; Terhart 1999), so dass man heute eher von eine konstruktivistischen Rahmung von Konzepte oder einem Bezug zu konstruktivistischen Überlegungen sprechen kann. Dieser Rahmen, den der Konstruktivismus liefern kann, hat ganz wesentliche Impulse für pädagogisches Denken und Handeln geliefert, - gleichwohl erweist sich die Ableitung wissenschaftlicher Aussagen für die Mediendidaktik als überraschend wenig eindeutig. Es handelt sich um didaktische Überlegungen, die in völlig anderen Zusammenhängen bereits formuliert und erprobt worden sind, der Bezug zu einem Konstruktivismus im engeren Sinne ist in der Regel kaum zwingend.

Für die Mediendidaktik stellt sich schliesslich die Frage, ob bei den genannten computertechnischen Umsetzungen tatsächlich von einer «Anwendung» konstruktivistischer Prinzipien auf das mediengestützte Lernen gesprochen werden kann. Oder handelt es sich hier nicht einfach um neue Softwaretechniken, die in den 90er Jahren den Weg zur multimedialen Informationsverarbeitung, zu anderen Benutzeroberflächen, einem anderen Umgehen mit Computern und einer veränderten gesellschaftlichen Konstruktion des Gegenstandes «Computer» markierten?

In der gesellschaftlichen Wahrnehmung digitaler Technik ist das System nicht mehr ein mit «Intelligenz» überhöhtes Gegenüber des Menschen, das den Lerner durch ein raffiniert programmiertes System führt und versucht, Dialoge zwischen Lernendem und Lehrendem nachzubilden. Das (netzbasierte) Computersystem wird zu einem Werkzeug, mit dem Lernende selbstständig Informationen aufrufen und auswählen und diese in verschiedener Weise alleine und mit anderen bearbeiten.

Der Übergang zum Konstruktivismus als Mainstream-Paradigma spiegelt also weniger eine theoretische Weiterentwicklung wider, sondern die ver- änderte gesellschaftliche Wahrnehmung und Nutzung von digitaler Technik in unserer Lebenswelt. Das heisst, die zunehmende Forderung nach z. B. Selbststeuerung beim computergestützten Lernen ist in gewisser Weise zunächst einfach Reflex darauf, dass Computer zunehmend steuerbar werden, und als zunehmend steuerbar erlebt und genutzt werden.

\section{Zur Bewertung des Konstruktivismus}

Das Problem für die Mediendidaktik sehen wir vor allem darin, dass das Label Konstruktivismus zu einer vagen Floskel wurde für etwas Neues, Positives, das sich von etwas Altem, Überkommenem absetzt, und sich für nahezu alle Lösungen strapazieren lässt. Hinzu kommt, dass relativ bald erkannt wurde, dass rein «konstruktivistische» Ansätze - ohne jede instruktionelle Komponente - in den meisten Fällen wenig problemadäquat sind, da sie gesellschaftliche Bildungsanliegen nicht hinreichend adressieren. So verwässerte der «gemässigt konstruktivistische» Ansatz durch Aufnahme instruktioneller Komponenten und fand den Weg in die weitgehende Beliebigkeit. Auf diese Weise ist der theoretische Fortschritt für die Mediendidaktik kaum befördert worden.

Überspitzt formuliert: Ein wesentlicher Vorzug des Labels Konstruktivismus wurde neben seiner positiven Aufladung seine Beliebigkeit. Typische Vertreter der klassischen Position, wie David Merrill, konnten sich mit marginalen Änderungen ihrer Aussagen dem neuen Label zuordnen (vgl. Merrill 1994). So ist es auch nicht verwunderlich, dass sich keine Publikationen finden, in der hinterfragt wird, ob bestimmte Lernanwendungen denn tatsächlich konstruktivistische Prinzipien erfüllen. Selbst bekannte Vertreter des Konstruktivismus scheuten nicht davor zurück, «konstruktivistisch inspirierte» Anwendungen vorzustellen, die die geforderten Prinzipien nur marginal umsetzen.

Die Karriere des Labels «Konstruktivismus» kann auch als ein sozialpsychologisch und wissenschaftssoziologisch interessantes Phänomen thematisiert werden: Die vielen Wissenschaftler/innen, die in den 90er Jahren in Medienprojekten plötzlich vor mediendidaktischen Entscheidungen standen, suchten nach einer Antwort auf die Frage, was ein «gutes» Lernmedium ausmacht. Der Konstruktivismus bot eine sozial akzeptierte Orientierung, in dem er eine Richtung aufzeigte, die positive Sanktionen versprach. Die Orientierung am «Konstruktivismus» fiel nicht schwer, das «Bekenntnis» blieb ungefährlich, weil es keine Gegner (mehr) gibt und es sichert die soziale Anerkennung innerhalb der scientific community, 
notfalls mithilfe der Versicherung, man würde der «gemässigten» Variante des Konstruktivismus zuneigen.

Der Konstruktivismus hat die theoretische Debatte der letzten Jahrzehnte in verschiedenen wissenschaftlichen Disziplinen geprägt. Kein anderer Begriff war gerade in der Mediendidaktik in den 90er Jahren so präsent wie dieser. Konstruktivismus wurde zur Chiffre für die Abkehr von Modellen des computergestützten Lernens, bei der die optimale Steuerung und Regelung des Lernprozesses seitens des technischen Systems angestrebt wird. Es rückten Ansätze in den Vordergrund, die die individuellen und sozialen Aktivitäten der Lernenden betonten, die Selbststeuerung des Lernprozesses ebenso wie den sozialen Kontext des Lernens.

Doch die damit einhergehende Vorstellung einer «paradigmatischen Überwindung» des Behaviorismus durch den Konstruktivismus muss aus Sicht der Mediendidaktik infrage gestellt werden. Der Mediendidaktik muss es um die Konzeption von Lernangeboten gehen, ihr muss daran gelegen sein, über ein möglichst breites Repertoire an methodischen Varianten in Abhängigkeit von Parametern des didaktischen Feldes zu verfügen. Die Vorstellung, dass es den einen besten Unterricht gibt, ist in der Allgemeinen Didaktik längst überwunden.

Aus Sicht der Mediendidaktik erscheint die Suche nach dem einen richtigen Paradigma überraschend fragwürdig, ja obsolet. Die Konstellationen, in denen mit Medien gelernt wird, sind so vielfältig, dass didaktische Prinzipien wie authentische Einbettung, Kooperation beim Lernen oder Lernen durch Lehren mögliche, aber nicht prinzipiell vorteilhafte Ansätze darstellen. Im Übrigen handelt es sich um grundlegende didaktische Prinzipien, die schon lange vor ihrer Überhöhung und vor allem völlig unabhängig vom Konstruktivismus in der Didaktik diskutiert wurden. ${ }^{2}$

\section{Der Pragmatismus als weiterführende Perspektive der Mediendidaktik}

Die bisherige - auf Lerntheorien fixierte - Diskussion in der Mediendidaktik ist vielfach mit einem meta-theoretischen Fehlschluss behaftet: Wenn man beispielsweise das Menschenbild und die epistemologischen

\footnotetext{
${ }^{2}$ Hier wäre bereits auch auf die Vorstellungen des US-amerikanischen Pragmatismus hinzuweisen und zwar insbesondere nach JOHN DEWEY: Er fasste Lernen als «Knowledge construction» auf und stellte den Kontextualismus in den Vordergrund seiner Überlegungen. Damit ist er verschiedentlich als «Vorläufer» der

konstruktivistischen Lerntheorie bezeichnet worden (Gerstenmaier/Mandl 1999b, S. 4; vgl. auch Clement/Kräft 2002).
}

$13 / 22$
Annahmen des Behaviorismus ablehnt, dann lehnt man auch entsprechende lerntheoretische Konzepte $a b$ und folglich auch die in diesem Zusammenhang prominent gewordenen Varianten zur (z. B. sequentiellen) Strukturierung von Lernmedien. Pointiert formuliert: Fühlt man sich etwa dem Menschenbild oder der Erkenntnistheorie des Konstruktivismus nahe, dann wird man beispielsweise einen problembasierten Ansatz des Lernens bevorzugen, sich deswegen für eine offene, hypermediale Lernumgebung entscheiden und stärker strukturierte Lernwege, vielleicht sogar mit Lernerfolgskontrollen, prinzipiell ablehnen.

Bei einer theoretischen Fundierung von Mediendidaktik kann es - unseres Erachtens - nicht darum gehen, das eine, richtige Paradigma des Lernens oder Lehrens zu identifizieren, da davon auszugehen ist, dass dieses als solches nicht existiert. Die zentrale Frage der Mediendidaktik lautet vielmehr, unter welchen Bedingungen Menschen wie mit Medien erfolgreich lernen können. Es geht darum den Prozess zu beschreiben, wie Lernmedien gestaltet werden können, um bestimmte Zielhorizonte zu erreichen. In der Beschreibung der Prozesse werden die vielfältigen Entscheidungsdimensionen sichtbar, die sich in einem solchen Gestaltungsproblem stellen («Didaktisches Design»).

Die Suche nach dem einen überlegenen, paradigmatischen Ansatz für das Lernen und Lehren hat die theoretische Weiterentwicklung der Mediendidaktik mehr blockiert als befördert. Es müsste vielmehr darum gehen, die Komplexität sozialer Realität anzuerkennen, und damit auch anzuerkennen, dass die Suche nach dem «one best way» für die Frage der Gestaltung von Lernangeboten irreführend ist. Diese Sicht entspricht der Position des Pragmatismus, der damit perspektivisch eine Basis für eine alternative theoretische Fundierung von Mediendidaktik bietet. Der Pragmatismus ist dabei keineswegs eine neue Modeströmung, die die bisherigen Paradigmen um eine neue Variante bereichert oder gar in Konkurrenz zu den bisherigen Ansätzen, etwa des Behaviorismus oder Konstruktivismus, tritt. Es handelt sich eher um einen Ansatz, der «quer» zu den bisherigen Konzepten liegt. Er bewertet die anderen Ansätze nicht als solches positiv oder negativ, sondern fragt jeweils in und für eine Situation, welches Konzept welchen Beitrag für eine Problemlösung liefert, die Perspektiven menschlichen Handelns und die Handlungsfähigkeit von Menschen erweitert.

Lerntheoretische Überlegungen alleine liefern für die Mediendidaktik keinen hinreichenden Rahmen, um die zentrale Frage der Zielproblematik, des Mehrwertes und des Nutzens ebenso wie der Implikationen des 
Medieneinsatzes für Bildung und Gesellschaft zu diskutieren. Auch die Spezifikation des Zielhorizontes auf der Ebene des Individuums bleibt unbefriedigend, da die Verbindung zu zentralen Kategorien, wie Medienkompetenz und -bildung, nicht hergestellt werden.

Mit der Fundierung der Mediendidaktik durch den Pragmatismus wird ein neuer und gleichzeitig alter Denkweg beschritten. Alt deswegen, weil der Pragmatismus als Wissenschaftstheorie und Grundlage einer Erziehungsphilosophie und Didaktik bereits seit der zweiten Hälfte des 19. Jahrhunderts vorliegt und mit den Namen Charles SANDERs PeIRCE, William James und John Dewey verbunden ist. Andererseits ist der Denkweg innovativ, weil er neben der Stabilität von Elementen der Lebenswelt den Kontingenzen und damit den Unwägbarkeiten und Unsicherheiten einer Situation den gleichen Stellenwert beimisst. Damit hebt er sich von traditionellen Vorstellungen ab, die den Anspruch formulieren, Kontingenzen zu überwinden, indem Konzepte mit universalen Ansprüchen formuliert werden, die Perspektiven zu (scheinbar) absoluter Sicherheit für den Menschen aufzeigen.

Der Pragmatismus (vom griech. «pragma»: das Getane; aber auch das, was zu tun ist; das Handeln, das Tun, die Tätigkeit) rückt Handlungen in das Zentrum der Betrachtung. JOHN DEWEY (1859 - 1952) geht von einem Gedanken aus, der für uns heute fast selbstverständlich klingt, nämlich dass Theorie und Praxis in einem engem Zusammenhang stehen. Er «zeigt, wie die Theorie sich aus der Praxis heraus entwickelt, wie die Theorie in der Form von Hypothesen zeitweise eine gewissen Selbständigkeit gegenüber der Praxis behauptet, um dann schliesslich Teil der Praxis zu werden, sich dort zu bewähren und in der Praxis eine Leitfunktion zu übernehmen ... Wenn immer möglich, beginnt der einzuschlagende Weg bei der Praxis und führt über die Theorie zur Praxis zurück» (Schäfer 1988, S. 5f).

Dass diesen Überlegungen des Pragmatismus verstärkt Aufmerksamkeit geschenkt wird, hängt mit der Debatte über die Postmoderne in den 80iger und 90iger Jahren ein Jahrhundert später zusammen. Die «postmoderne» Zeit ist gekennzeichnet durch eine Verunsicherung über die Fortschrittsverheissungen der Moderne und die Ideale der Aufklärung. Erneut wird herausgestellt, dass menschliches Handeln, Denken und Fühlen immer nur im Zusammenhang mit der jeweiligen Situation zu verstehen sind. Auch für das menschliche Lernen gibt es keinen «goldenen» Königsweg, der nicht abhängig wäre von zeitlichen, kulturellen und situativen Bedingungen.

Der Pragmatismus entstand, als die Industriegesellschaft mit ihren gesell-

$15 / 22$ schaftlichen Verwerfungen heraufzog und damit - auch von Seiten der Wissenschaft - verstärkt nach praktischen Problemlösungen verlangt wurde. Die Bedeutung sekundärer, von Anderen vermittelter Erfahrungen nahm gegenüber primären, unmittelbaren Erfahrungen zu. Zwischenmenschliche Beziehungen wurden zunehmend als nachhaltig gestört erlebt. Nicht umsonst hebt DEWEY den Demokratiegedanken und die Kommunikation in seinen Werken hervor. ${ }^{3}$ Als einer der Gründungsväter des Pragmatismus hat er den Ansatz des Pragmatismus in den pädagogischen Kontext eingebracht.

\section{Aussagen des Pragmatismus zur Medienbildung}

Zur theoretischen Fundierung von Mediendidaktik aus Sicht des Pragmatismus sollen im Folgenden Grundaussagen einer Theorie der pragmatistischen Medienbildung vorgestellt werden. Zunächst beinhaltet «Medienbildung» mit den Begriffen «Medien» und «Bildung» zwei Begriffe, die in bestimmter Weise miteinander verknüpft sind: Zum einen geht es darum, wie Medien zu Bildung beitragen können («Medien für Bildung»). Es stellt sich die Frage, wie Medien zu Zwecken des Lernens und Lehrens mit einer bestimmten methodischen Aufbereitung und verschiedenen Zielhorizonten eingesetzt werden können.

Gleichzeitig geht es «Medienbildung» aber auch um die Frage, wie Bildung zur Bewältigung einer Medien- und Wissensgesellschaft beitragen kann («Bildung für Medien»). Dabei geht es aus Sicht des Pragmatismus nicht um bestimmte, von einer Theorie vorgebbare Bildungsinhalte und -ziele, wie sie in den vielen Katalogen einer «Medienkompetenz» formuliert werden. Das Ziel ist vielmehr allgemeiner zu formulieren; es geht darum, Menschen teilhaben zu lassen an der hochgradig mediatisierten Kultur und Gesellschaft.

Dabei sind die beiden Aspekte «Medien» und «Bildung» als miteinander verschränkt zu sehen: Die Nutzung von Medien für Bildungszwecke sollte genau auch die Teilhabe an Kultur und Gesellschaft fördern, und die Bildung für die Medien- und Wissensgesellschaft sollte sinnhafter Weise eben auch die Medien nutzen, um diese Bildungsprozesse zu ermöglichen.

Anders als bei vielen bisherigen Bildungstheorien kann es aus Sicht einer

${ }^{3}$ Mittlerweile gibt es eine Reihe von Ausdifferenzierungen des amerikanischen Pragmatismus: z. B. den methodologischen, semantischen und Fundamentalpragmatismus. An der Weiterentwicklung des Pragmatismus sind u. a. auch Quine, Sellars, Davidson, Putnam und Rorty beteiligt (vgl. Sandbothe 2000). 
pragmatistisch begründeten Bildungstheorie nicht darum gehen, ideale (Bildungs-) Ziele, die es anzustreben gilt, vorzugeben. An die Stelle solcher, von aussen gesetzter Ziele betont DEWEY den relationalen Charakter von Zielen, Mitteln und Konsequenzen im Prozess der Erfahrung. Damit ist gemeint, dass Ziele, Mittel und Konsequenzen nie für sich gesehen und gewertet werden können, sondern nur im Zusammenhang (in «Relation») zu der Situation zu verstehen sind, in der sich Menschen befinden. DEwEY hängt dabei keineswegs einem Relativismus an, der jedes Ziel in gleicher Weise und gleichermassen akzeptieren würde. Es ist vielmehr so, dass eine Bewertung nicht a priori, von aussen gegeben werden kann, sondern einen bestimmten Prozess erforderlich macht.

Der pragmatistische Denkansatz setzt an Situationen an, die Menschen erleben und typischerweise durch Kontingenzen geprägt sind. Er begreift diese Unsicherheiten und Unwägbarkeiten von Situationen nicht als notwendiges Übel, das es zu überwinden gilt. Im Gegenteil: Die Auseinandersetzung mit diesen Kontingenzen begründet vielmehr die Freiheit menschlichen Handelns, sich so oder auch anders zu entscheiden, und damit bildende Erfahrungen

Menschen stellen in einem Prozess der Erfahrung in einer bestimmten Situation - rückwärts wie vorwärts gewandt - Bedeutungen zwischen Zielen, Mitteln und Ergebnissen ihres Handelns her. «Es bedeutet, dass die Erfahrung als ein aktiver Vorgang ... Zeit erfordert, und dass die späteren Teile dieses Vorgangs die früheren vervollständigen; die späteren Teile bringen Beziehungen ans Licht, die in den früheren bereits vorhanden und wirksam, aber noch nicht erkannt waren» (Dewey 2000, S. 111). Es handelt sich hier um einen Prozess, in dem Erfahrungen rekonstruiert werden. Dieser Prozess der Rekonstruktion von Erfahrung ist gleichsam eine Methode mit Realität umzugehen und auch zentraler Bestandteil des pragmatistischen Bildungsverständnisses. Es resultiert letztlich daraus, dass es der Pragmatismus ablehnt, Lern- und Erfahrungsinhalte zu bewerten.

Eine Bildungstheorie kann heute kaum mehr einen festen Kanon von Bildungsinhalten vorgeben, sondern muss methodische Wege beschreiben, wie Menschen bildende Erfahrungen machen können. Letztlich geht es darum, Handlungsmöglichkeiten und Handlungsfähigkeiten von Personen in einer Medien- und Wissensgesellschaft, die eine ständige, lebenslange Aneignung und Weiterentwicklung von Wissen fordert, zu erweitern. Diese methodische Fähigkeit avanciert zu einer Schlüsselkompetenz zur Teilhabe an Kultur und Gesellschaft.
So gestaltet sich aus dieser Perspektive Medienbildung als ein inhaltlich ausgerichteter, nie abgeschlossener Prozess der Erfahrung von Menschen in Auseinandersetzung mit ihrer (medialen) Umwelt. Dieser Erfahrungsprozess ist mehr als ein «Erfahrungslernen», das zufällig - als Ergebnis von privaten «Erlebnissen»- geschieht. Nicht jedes Erlebnis wird demnach zu einer «bildenden Erfahrung». Hierzu ist erforderlich, dass Erlebtes reflektiert und auf das eigene Handeln bezogen wird bzw. Konsequenzen für das eigene Handeln hat. Diese Erfahrungen sind dadurch gekennzeichnet, dass sie sich ständig im Fluss befinden, sie bauen auf vorliegenden Erfahrungen auf, ordnen Erfahrungen neu und werfen Perspektiven für zukünftiges Handeln auf. Bei dem Prozess der Bildung handelt es sich um «eine ständige und kontinuierliche Rekonstruktion von Erfahrung, ein(en) dauernde(n) Neuaufbau, eine ständige Reorganisation» (Dewey 2000, S. 75). Prozess und Ziel der Bildung sind ein und dieselbe Sache, der Prozess der Erfahrung ist identisch mit dem Ergebnis von Erfahrung.

Eine pragmatistische Theorie von Medienbildung geht damit nicht von idealisierten Bildungszielen aus, sondern von menschlichen Situationen, die in ihrer Singularität Kontingenzen, Zufälligkeiten und unvorhersehbare Möglichkeiten einschliessen und dadurch dem Menschen einen Spielraum für sein Handeln eröffnen. Über die Reflexion kann er der Instabilität und Kontingenz von Welt in «Erfahrungen» begegnen und sich so in komplexen, mediatisierten Umwelten zurechtzufinden.

Führt man diese Betonung des Situativen fort, so ergibt sich eine weitere Forderung: Wenn man die Komplexität von Situationen anerkennt, dann erscheint ein Denken in Dualismen, wie es in anderen Bildungstheorien vorherrscht, fragwürdig. ${ }^{4}$ Ein Denken in Relationen dagegen entspricht eher der Offenheit von Welt. Das Denken in Relationen lässt zu, dass Unterschiede gewahrt bleiben und gleichzeitig Verbindungen und Übergänge bedacht werden. Es ist die Konsequenz daraus, dass der situative Handlungsbezug die Grundlage des Erfahrungsprozesses ist (vgl. de Witt 1999). Gerade in unserer hochgradig vernetzten Welt mit ihren vielen Querbezügen und Abhängigkeiten erscheint ein Denken in universalen Ansprüchen obsolet.

Lernen meint dann «dem, was um uns herum passiert und in uns passiert, Bedeutung (meaning, significance) zu geben. Lernen ... ist eine Tätigkeit,

\footnotetext{
${ }^{4}$ Z. B. der Dualismus zwischen «Körper» und «Geist» oder «Freiheit» und «Unfreiheit», aber auch zwischen «echten, hochwertigen» Bildungszielen und «trivialen, einfachen» Qualifizierungsinhalten.
} 
durch die das, was um uns herum und in einem passiert, verständlich wird» (Meyer 1999, S. 57). Eine pragmatistisch orientierte Mediendidaktik setzt sich also mit erfahrungsgeleitetem Lernen mit medialen Angeboten und in mediatisierten Umwelten auseinander. Dabei wären mediendidaktische Entscheidungen auf das Strukturmuster des Prozesses des «Inquiry» auszurichten, das Dewey immer weiter ausdifferenziert und 1916 in seinem Werk «Demokratie und Erziehung» als «Stufen des Denkens» beschrieben hat. $^{5}$

\section{Fazit}

Der Pragmatismus bietet für die Mediendidaktik eine Denkfolie, auf deren Hintergrund die theoretische Fundierung von Mediendidaktik anders bzw. besser erfolgen kann.

Die zentrale Konsequenz einer solchen Fundierung von Mediendidaktik durch den Pragmatismus lautet: Eine Situation bestimmt den Wert eines Mediums, und nicht das Medium selbst. Medien sind Artefakte, die keinen Wert an sich haben, sondern ihre Bedeutung erhalten sie erst durch eine bestimmte Nutzung von Menschen in bestimmten Kontexten und zu bestimmten Zeiten. Bei der Gestaltung mediengestützter Lernangebote ist deswegen die gesamte Lernorganisation einschliesslich der Einbettung eines Angebotes in ein soziales System zu berücksichtigen.

Lernende sind z. B. in Aktivitäten zu verstricken, bei denen sie die Konsequenzen ihres Handelns antizipieren lernen und verantwortungsvolle Entscheidungen treffen - sei es durch entsprechende Lern- und Übungsaufgaben, Kommunikationsformen, das Eingebundensein in Interaktionssituationen. Es kommt darauf an, Lern- und Denkwege und -

5 «Wir können zwar mit Recht von der `Methode des Denkens`sprechen; wichtiger ist es jedoch zu wissen, dass das Denken die Methode der bildenden Erfahrung ist. Die wesentlichen Merkmale der 'Methode` sind darum identisch mit den wesentlichen

Merkmalen des `Denkens`: ...erstens, dass der Schüler eine wirkliche, für den Erwerb von Erfahrung geeignete Sachlage vor sich hat - dass eine zusammenhängende

Tätigkeit vorhanden ist, an der er um ihrer selbst willen interessiert ist; zweitens: dass in dieser Sachlage ein echtes Problem erwächst und damit eine Anregung zum

Denken; drittens: dass er das nötige Wissen besitzt und die notwendigen

Beobachtungen anstellt, um das Problem zu behandeln; viertens: dass er auf mögliche Lösungen verfällt und verpflichtet ist, sie in geordneter Weise zu entwickeln;

fünftens: dass er die Möglichkeit und die Gelegenheit hat, seine Gedanken durch

praktische Anwendung zu erproben, ihren Sinn zu klären und ihren Wert selbständig zu entdecken» (Dewey 2000/ 1916, S. 218).

$19 / 22$ werkzeuge zu öffnen, um eigenständig mit Pluralitäten, Kontingenzen, neuen Erfahrungen einer komplexen, mediatisierten Umwelt umzugehen. Lernende sollen Vorstellungen entwickeln, wie Handlungen zur Lösung von Problemen führen. Dazu müssen sie lernen, die Konsequenzen von Handlungen zu antizipieren und neue Erfahrungen mit vergangenen Erfahrungen in Verbindung bringen.

Medienbildung, die sich an dem Ansatz des Pragmatismus ausrichtet, kann dem Einzelnen eine Orientierung in einer Gesellschaft geben, die zunehmend hohe Ansprüche im Hinblick auf Vielfältigkeit, Flexibilität, Spontaneität und Leistung stellt. Mit der Forderung nach selbstgesteuertem und lebenslangem Lernen wird ein Bildungsbegriff anvisiert, der davon ausgeht, dass vom Einzelnen eine ständige Rekonstruktion medialer Erfahrungen und Wissensstrukturen verlangt wird. Lernen findet dabei nicht allein in den Begriffen der Handlung oder nur in Begriffen des Inhalts statt. Lernen wird über die Handlung als authentische Aktivität definiert, die in Relation zu dem Inhalt steht. Der Lernprozess beinhaltet dann z. B. die Fähigkeit kritisch in den Inhaltsbereichen zu denken, Informationen zu beschaffen, die in Relation zu einem Problem stehen und sie dann über eine Reflexionsphase zur Geltung zu bringen. Aus Überlegungen des Pragmatismus wäre etwa zu fordern, dass eine einseitige Theorievermittlung oder eine beliebige Praxisorientierung vermieden wird.

Die Perspektive des Pragmatismus wirkt sich bei didaktischen Entscheidungen schliesslich auch darin aus, dass «Hierarchisierungen», soweit es geht, aufzulösen versucht wird: Lernziele und -mittel sind immer jeweils von der aktuellen Lernsituation aus zu denken und von hier in Beziehung zueinander zu sehen. Eine entsprechend durch den Pragmatismus fundierte «gestaltungsorientierte» Mediendidaktik ist darauf auszurichten, Bildungsanliegen und -probleme zu lösen und nicht nur auf die sich ständig ändernden, kurzfristigen Qualifikationsanforderungen der Gesellschaft zu reagieren.

Mit der Berücksichtigung dieser Überlegungen des Pragmatismus gewinnt die Mediendidaktik eine Möglichkeit, Kriterien für die Frage der Lehrinhalte und -ziele bei der Gestaltung von Lernangeboten $\mathrm{zu}$ formulieren. Für die Weiterentwicklung der Mediendidaktik wird es notwendig sein, die Konzepte und Dimensionen von Medienbildung in der Mediendidaktik als qualitätssichernden Erfahrungs- und Gestaltungsprozess stärker einzubringen. Bildungsziel ist daher letztlich die Partizipation von Menschen an der Gestaltung der Medien- und Wissensgesellschaft. 
MedienPädagogik

\section{Literatur}

Anderson, J. R./ Boyle, C. F./ Reiser, B. J. (1985): Intelligent tutoring systems. Science, 228, S. 456-462.

Benjamin, J. (1988): A history of teaching machines. American psychologist, 43, S. 703-712.

Clement, U./ Kräft, Kl. (2002): Lernen organisieren. Medien, Module, Konzepte. Berlin.

Cube von, F. (1980): Die kybernetisch-informationstheoretische Didaktik (Didaktisches Forum III). Westermanns Pädagogische Beiträge, S. 121-124.

Dewey, J: (1995): Erfahrung und Natur. Frankfurt (LW1:1925).

Ders. (2000): Demokratie und Erziehung. Weinheim (MW9:1916).

Dick, W. (1987): A history of instructional design and its impact on educational psychology. In: Glover, J. A./ Ronning, R. R. (Hrsg.): Historical foundations of educational psychology. New York, S. 183-202.

Dubs, R. (1995): Konstruktivismus: Einige Überlegungen aus der Sicht der Unterrichtsgestaltung. Zeitschrift für Pädagogik, 41, S. 889-903.

Duit, R. (1995): Zur Rolle der konstruktivistischen Sichtweise in der naturwissenschaftlichen Lehr- und Lernforschung. Zeitschrift für Pädagogik, 41, S. 905-923.

Frank, H. (1996): Bildungskybernetik. München.

Frank, H./ Meder, B. S. (1971): Einführung in die kybernetische Pädagogik. München.

Gerstenmaier, J./ Mandl, H. (1995): Wissenserwerb unter konstruktivistischer Perspektive. Zeitschrift für Pädagogik, 41, S. 867-888.

Gerstenmeier, J./ Mandl, H. (1999a). Konstruktivistische Ansätze in der Erwachsenenbildung und Weiterbildung. In: Tippelt, R. (Hrsg.): Handbuch Erwachsenenbildung/Weiterbildung. Opladen.

Gerstenmaier, J./ Mandl, H. (1999b): Konstruktivistische Ansätze in der Erwachsenenbildung und Weiterbildung. Forschungsbereicht München.

Glasersfeld, E. v. (1999): Konstruktivismus und Unterricht. Zeitschrift für Erziehungswissenschaft, 2(4), S. 499-506.

Hilgard, E. R. (1964): Theories of learning and instruction (63rd Yearbook of the NSSE). Chicago.

Mandl, H./ Lesgold, A. (Hrsg.) (1988): Learning issues for intelligent tutoring systems. Berlin.

Merrill, M. D. (1994): Instructional design theory. Englewood Cliffs, NJ.
Meyer, M. A. (1999): Problemlösendes Lernen in der Pädagogik John Deweys. In: Pädagogik 10/99, 29-32.

Pagliaro, L. A. (1983): The history and development of CAI: 1926-1981, an overview. Alberta journal of educational research, 29, 75-84.

Polson, M. C./ Richardson, J. J. (1988): Foundations of intelligent tutoring systems. Hillsdale, NJ.

Reiser, R. A. (1987). Instructional Technology: A History. In: Gagné, R. M. (Hrsg.): Instructional technology: Foundations. Hillsdale, NJ, S. $11-48$.

Rustemeyer, D. (1999). Stichwort: Konstruktivismus in der Erziehungswissenschaft. Zeitschrift für Erziehungswissenschaft, 2(4), 467-484.

Sandbothe, M. (Hrsg.) (2000): Die Renaissance des Pragmatismus. Weilerswist.

Schäfer, K.-H. (1988): Franz Fischers Bildungskategoriale Pädagogik und die Interaktionspädagogik. In: Franz-Fischer-Kreis: Norderstedter Hefte für Philosophie und Pädagogik, 3. Jg., Heft 1/ 2, S. 3-26.

Siebert, H. (1999). Pädagogischer Konstruktivismus. Eine Bilanz der Konstruktivismusdiskussion für die Bildungspraxis. Neuwied.

Sleeman, D./ Brown, J. S. (1982). Intelligent tutoring systems. London.

Terhart, E. (1999). Zum Konstruktivismus als alternatives Paradigma der Didaktik. Zeitschrift für Pädagogik.

de Witt, C. (1999): Neue Medien und die Pädagogik des Pragmatismus. Dortmund. 